\title{
Aspectos históricos de la Enfermedad Trofoblástica Gestacional
}

\author{
Miguel Eduardo Aragón*
}

La Enfermedad Trofoblástica gestacional (ETG), comprende un alto espectro de entidades, entre ellas, la Mola Hidatidiforme (Completa, Parcial), Mola invasora y coriocarcinomia; todas ellas derivadas de la placenta humana, caracterizadas con mucha exactitud en la actualidad gracias a los avances de la Inmunohistoquímica, la genética y la bioquímica aplicada.

Los autores griegos y romanos, hacían referencia a las Molas sin distinguir entre sanguíneas, carnosas y vesiculares (1).

Rossmann, Hipócrates y su discípulo Diokles consideraban a las Molas como una degeneración mixomatosa del corion ( Cos, año 400 a.c.) (1).

Aetius de amidas, en el siglo VI médico de la corte de Justimano I, da el nombre de "gota de agua", del griego hydatis que significa gota (2).

El español Cristóbal de la Vega, en el liber de arte medendi, publicado en Lyon en 1564 dice : "de la fusión de los humores del semen sobre el útero se desarrollaba unas vejigas desprendidas dentro del mismo, las cuales están llenas de un humor acuoso y las mujeres creen que han concebido, pues el vientre se inflama y se retiene la menstruación", agrega a título de caso clínico, y doña Leonor de Zantaren expulsó vejigas membranosas y otras membranas que juntas pesaron 12 libras romanas al cabo de seis meses de gestación e innumerables molestias (1).

Anécdota similar, la sucedida a la condesa de Henneberg que por maldición dio a luz 365 "niños muertos" el viernes santo de 1276 (3).

En 1700 William Smellie recopila la descripción morfológica más completa hasta este momento con notoriedad los términos Mola Hidatidica.

En el siglo XVIII (1782), Goetze, suponía que la Mola Hidatidica (MH) era producida por gusanos parásitos, hipótesis reactivada hace unos años con el señalamiento del helminto "Hidatoxi Lualba".

En el siglo XIX ( 1827 ), Velpeau, retoma la descripción hipocrática: degeneración de las vellosidades coriales o su equivalente, la dilatación quística de la vellosidad coriónica.

Virchow (1863) La MH es una transformación gelatinosa y tumoral del estroma (tumor mixomatoso), controvertida por Storch, quien consideraba que no era un

Profesor Asociado. Departamento de Ginecología Obstetricia. Facultad de Medicina. Universidad Nacional. Servicio Oncología Ginecológica - IMI. 1997 tumor sino una hiperplasia del corion con degeneración quística secundaria.

Sanger, (1879) sostenía que el tumor derivaba de la decidua del embarazo, al paso de esta aseveración salió Eva Chaletzky y posteriormente Marchand (1895), cuya descripción aun tiene vigencia al negarle a la $\mathrm{MH}$ la propiedad tumoral pero caracterizándola por el aumento de los fenómenos de degeneración y proliferación en el revestimiento epitelial de las vellosidades -sincitio y células de Langhans- al mismo tiempo que se origina la fluidificación (edema) dêll estroma, causa de transformación quística de las vellosidades.

Jhon Teacher (1903), de Glasgow, amplió el trabajo de Marchand sobre la etiología epitelial y propuso una clasificación (5).

Stoeckel, sugiere que los quistes Teca-luteinicos son la etiología de la MH. La discusión continua, llegándose a creer que hay alguna relación genética entre los dos procesos ( $\mathrm{MH}$ y quistes), aparecen con la enfermedad y desaparecen con el tratamiento de la MH (6), explicada hoy por la estimulación de la HCG al ovario, concomitante con los altos valores de la hormona, fruto de la excesiva proliferación trofoblástica (5).

Respecto a la histopatogénesis, Hertig y Edmonds (1940) plantean la hipótesis que se inicia con la muerte del embrión, seguida por edema intravellositario e hiperplasia trofoblástica, contraria a la hipótesis de Park (1959), en donde las anomalías estructurales del trofoblasto son las que en ultimas causan la muerte del embrión. De Hertig se conoce la sentencia "God's first cancer and man's first cure" (Primer cancer de Dios y la primera curación del hombre) $(3,5)$.

Szulman, resume el conocimiento de la enfermedad entre las décadas del 70 al 80 diciendo : "el trofoblasto es un tejido único que se origina a partir de la masa celular exterior del embrión antes de la implantación, es decir, se encarga de la fijación física en el endometrio y la secreción de la HCG, que detiene la menstruación y perpetúa el embarazo. Todo lo anterior basado en las propiedades pseudomalignas de la placenta humana argumentada en 3 propiedades fundamentales : 1.- privilegio inmunoligo, por encontrarse el trofoblasto desprovisto de los antígenos de transplante (HLA y ABO), evitando así el rechazo inmunológico. 2.- Invasividad, y 3.- Proliferación celular acelerada (hiperplasia) (7).

Hacia 1970, 2 grupos de trabajo japoneses (Kajii y Ohama 1977 ; Wake y Sasaki 1978), formularon una 
excitante observación: "la MH completa contiene cromosomas pares derivados del padre (diploidia diandrica), y la $\mathrm{MH}$ parcial o incompleta (triploidia diandrica), es decir, la estructura placentaria es genotípicamente del padre, y la estructura embrionaria o fetal es genotípicamente de la madre (7) acabando con el concepto de MH transicional.

Fernando Sánchez Torres, en su libro Historia de la Ginecobstetricia en Colombia (1993), cita su estudio, en donde demuestra que los cambios histopatológicos del riñón, propios de la preeclampsia-eclampsia tardía, eran exactamente iguales a los observados en la forma temprana asociada a la $\mathrm{MH}(8)$.

\section{Hormona coriónica gonadotrópica ( HCG )}

Es el marcador tumoral ideal en la ETG, lo que ha permitido un gran avance en la monitoría del seguimiento terapéutico y la formulación del prognóstico de su evolución biológica.

En 1928 Ascheim y Zondek, aislaron una sustancia en la orina de la mujer embarazada que estimula las gónadas en humanos y animales.

En 1930 cultivos tisulares confirmaron el origen trofoblástico de la hormona y "se llamó HCG. Wide y Gemzelle, demostraron la formación de un anticuerpo contra la aplicación inyectable de HCG lo que permitió el desarrollo de inmunoensayos para medir los niveles de la Hormona. La combinación de técnicas inmunológicas e isotópicas por Berson y Yelow facilitó el camino para el desarrollo del RadioInmunoEnsayo (RIA) y la caracterización química de la subunidades de la HCG y el posterior desarrollo de RIA altamente específicos para cada una de estas subunidades.

Vaitukaitis y colaboradores del INS de los E. U. idearon la prueba para medir la subunidad B. Vinieron pruebas altamente especificas con variedad de técnicas., así : a) IRMA, prueba inmunoradiométrica, en donde el anticuerpo que se encuentra en exceso se marca. B) ELISA, Inmunovaloración ligada a enzima, en donde el agente marcador isotópico ha sido sustituido por una enzima. C) FIA, o método de fluoroinmunovaloración, que mide fotones a partir de marcaciones fluorescentes, por ejemplo DELFIA-HCG. Durante algún tiempo se midieron con anticuerpos monoclonales las fracciones libres de las subunidades alfa y Beta, en función del pronóstico de la ETG.

\section{Epoca terapéutica}

Antes de 1958, la ETG metastásica era mortal en un $98 \%$, en los primeros 6 meses de hecho su diagnostico, independiente del tratamiento aplicado (cirugía y radioterapia). Decía el Dr. F. B. Carter "si en una paciente se diagnostica coriocarcinoma metastásico y sobrevivió, debe dudarse en forma importante la precisión del diagnostico".

Hertz, en 1948, observó que la administración de ametopterina, un compuesto antifólico inhibía el crecimiento celular inducido por estrógenos, y agregando Acido fólico en exceso a la dieta de recobraba el crecimiento celular inicial.

Evans y colaboradores observaron que el Metotrexate (MTX) inducía lesio y reabsorción fetal en ratas. LI, en 1954, trató una paciente con MTX, con diagnostico de Melanoma y valores altos de HCG. Hertz, en 1956, en el Instituto Nacional de Salud de los E.U. en la ciudad de Bethesda, curó una paciente con diagnóstico de coriocarcinoma metastásico al administrar MTX. Posteriormente se usó el antibiótico Actinmicina D y el Clorambucil o ciclofosfamida, dando origen al tratamiento triconjugado conocido con la sigla MAC, para pacientes con ETG de alto riesgo. Con asombro, estas pacientes podían embarazarse nuevamente y dar frutos normales!.

La combinación de MTX y Acido folinico en 1961 hizo notoria la disminución de la toxicidad el agente antifólico (Bagshawe). El régimen EMA-CO (Etoposido, MTX, Actinomicina D, ciclofosfamida y Vincristina) curó el $83 \%$ de las pacientes con metástasis y alto riesgo de la ETG (1984), Surwit en 1991 sustituye el Etoposido por Cisplatino.

En Resumen, 3 hechos enmarcan el éxito del conocimiento de la ETG :

1.- Los progresos en Inmunología, genética y patología, cuya adecuada aplicación, ha permitido precisar y definir la enfermedad.

2.- La aparición de pruebas bioquímicas para medir la HCG, que con la implementación de modernas herramientas tecnológicas (RIA, IRMA, ELISA, DELFIA), hizo posible convertir esta hormona en el marcador ideal de la ETGm vital para el seguimiento de las pacientes en Observación o Tratadas.

3.- La espectacular respuesta a la quimioterapia, logrando dividir el tratamiento de la enfermedad en dos ERAS, hoy curable hasta en un $90 \%$ en situaciones clínicas clasificadas como metastásica de alto riesgo.

\section{BIBLIOGRAFIA}

1. Nubiola P. y Zarate E. Tratado de Obstetricia. Tomo II . Editorial Labor S.A. 1951.

2. Carter J. et al. An Update on gestational Trophoblastic Disease. Postgraduate Obstetrics and gynecology. 1991 ; 11(8): 1-8.

3. Hammond B.C. Neoplasias Trofoblásticas Gestacionales :Historia de los conocimientos actuales. Ginecología y Obstetricia. Temas Actuales. Vol. 31988

4. Disaia P. and Creasman W. Gestational Trophoblastic Newoplasia. In clinical Gynecologic Oncology 1997; 180. Mosby.
5. Gómez P. y cols. : Enfermedad Trofoblástica Gestacional. Rev. Col. Obst. Ginec. 1972; 23 (6): 469-501.

6. Stander H. J. Hydatiform Mole. In Text Book of Obstetrics. 1945 .3a. Revision. New York.

7. Redman C.W.G. The Human placenta. 1993. Oxford. Blackwell Scientific Publications.

8. Sánchez F. Historia de la Ginecobstetricia en Colombia. 1993. 1a. Edición. Santafé de Bogotá. 